\title{
On a Tree and a Path with No Geometric Simultaneous Embedding ${ }^{\star}$
}

\author{
Patrizio Angelini ${ }^{1}$, Markus Geyer ${ }^{2}$, Michael Kaufmann ${ }^{2}$, and Daniel Neuwirth ${ }^{2}$ \\ 1 Dipartimento di Informatica e Automazione - Università Roma Tre, Italy \\ angelini@dia.uniroma3.it \\ ${ }^{2}$ Wilhelm-Schickard-Institut für Informatik - Universität Tübingen, Germany \\ geyer/mk/neuwirthainformatik.uni-tuebingen.de
}

\begin{abstract}
Two graphs $G_{1}=\left(V, E_{1}\right)$ and $G_{2}=\left(V, E_{2}\right)$ admit a geometric simultaneous embedding if there exists a set of points $P$ and a bijection $M: P \rightarrow V$ that induce planar straight-line embeddings both for $G_{1}$ and for $G_{2}$. The most prominent problem in this area is the question whether a tree and a path can always be simultaneously embedded. We answer this question in the negative by providing a counterexample. Additionally, since the counterexample uses disjoint edge sets for the two graphs, we also prove that it is not always possible to simultaneously embed two edge-disjoint trees. Finally, we study the same problem when some constraints on the tree are imposed. Namely, we show that a tree of height 2 and a path always admit a geometric simultaneous embedding. In fact, such a strong constraint is not so far from closing the gap with the instances not admitting any solution, as the tree used in our counterexample has height 4 .
\end{abstract}

\section{Introduction}

Embedding planar graphs is a well-established field in graph theory and algorithms with many applications. Keystones in this field are the works of Thomassen [18], Tutte [19], and Pach and Wenger [17], dealing with planar and convex representations of graphs.

Recently, motivated by the need of concurrently represent different relationships among the same elements, a major focus in the research lies on simultaneous graph embedding, in which, given a set of graphs with the same vertex-set, the goal is to place the vertices on the plane so that all the graphs are planar, when drawn separately. Problems of this kind frequently arise in the visualization of evolving networks and in the visualization of huge and complex relationships, as the graph of the Web.

Among the many variants of this problem, the most important and natural one is the geometric simultaneous embedding (GSE). Given two graphs $G_{1}=\left(V, E^{\prime}\right)$ and $G_{2}=\left(V, E^{\prime \prime}\right)$, the task is to find a set of points $P$ and a bijection $M: P \rightarrow V$ that induce planar straight-line embeddings for both $G_{1}$ and $G_{2}$.

In the seminal paper on this topic [3], Brass et al. proved that GsEs of pairs of paths, of cycles, and of caterpillars always exist. A caterpillar is a tree such that deleting all its leaves yields a path. On the other hand, they provided negative results for a pair of

\footnotetext{
* This work was supported in part by MIUR of Italy, project AlgoDEEP prot. 2008TFBWL4, and by the German Research Foundation (DFG), project KA 812/15-1 'Graph Drawing for Business Processes'.
} 
outerplanar graphs and for three paths. Erten and Kobourov [6] found a planar graph and a path not allowing any GSE. Geyer et al. [14] proved that there exist two edge-disjoint trees not admitting any GSE. Finally, Cabello et al. [4] showed a planar graph and a matching not admitting any GSE and gave algorithms to obtain a GSE of a matching and a wheel, an outerpath, or a tree. The most important open problem is the question whether a tree and a path always admit a GSE or not, that is the subject of this paper.

Many variants of the problem, where some constraints are relaxed, have been studied. In the simultaneous embedding setting, where the edges do not need to be straight-line segments, any number of planar graphs admit a simultaneous embedding, since any planar graph can be planarly embedded on any given set of points in the plane [1617]. However, the same does not hold in the simultaneous embedding with fixed edges setting [11|138], in which the edges shared by the two graphs have to be represented by the same Jordan curve. Finally, the research on this problem opened a new exciting field of problems and techniques, like ULP trees and graphs [7 9[10], colored simultaneous embedding [2], near-simultaneous embedding [12], and matched drawings [5], deeply related to the general fundamental question of point-set embeddability.

In this paper we study the GSE problem of a tree and a path. We answer the question in the negative by providing a counterexample, that is, a tree and a path not admitting any GSE. Moreover, since the tree and the path used in our counterexample do not share any edge, we also negatively answer the question on two edge-disjoint trees.

The main idea is to use the path to enforce part of the tree to be in a non-planar configuration. Namely, we consider level nonplanar trees [7]10], that is, trees not admitting any planar embedding if their vertices must be placed inside certain regions according to a particular leveling. The tree of the counterexample contains many copies of such trees, while the path creates the regions. To prove that at least one copy has the non-planar leveling, we need a huge number of vertices, which is often needed just to ensure the existence of certain structures playing a role in our proof. A much smaller counterexample could likely be constructed with the same techniques, but we decided to prefer the simplicity of the arguments rather than the search for the minimum size.

In Sect. 2 we give define level nonplanar trees. In Sect. 3 we describe a tree $\mathcal{T}$ and a path $\mathcal{P}$, and in Sect. 4 we show that $\mathcal{T}$ and $\mathcal{P}$ do not admit any GSE. In Sect. 5 we give an algorithm to construct a GSE of a tree of height 2 and a path and in Sect. 6 we make some final remarks. Omitted proofs can be found in the full version of the paper [1].

\section{Preliminaries}

A (undirected) $k$-level tree $T=(V, E, \phi)$ is a tree $T^{\prime}=(V, E)$, called the underlying tree of $T$, together with a leveling of its vertices given by a function $\phi: V \mapsto$ $\{1, \ldots, k\}$, such that for every edge $(u, v) \in E$, it holds $\phi(u) \neq \phi(v)$ (see [7]10]). A drawing of $T=(V, E, \phi)$ is a level drawing if each vertex $v \in V$ such that $\phi(v)=i$ lies on a horizontal line $l_{i}=\{(x, i) \mid x \in \mathbb{R}\}$. A level drawing of $T$ is planar if no two edges intersect except, possibly, at common end-points. A $k$-level tree is level nonplanar if it does not admit any planar level drawing. We extend this concept to the one of region-level drawing by enforcing the vertices of each level to lie inside a region rather than on a horizontal line. Let $l_{1}, \ldots, l_{k}$ be $k$ non-crossing straight-line segments and let $r_{1}, \ldots, r_{k+1}$ be the regions such that any straight-line segment connecting a 


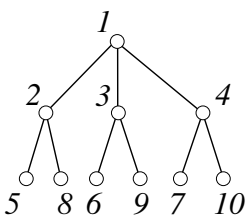

(a)

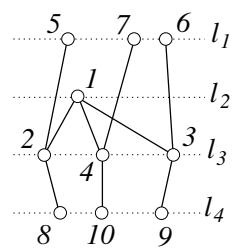

(b)

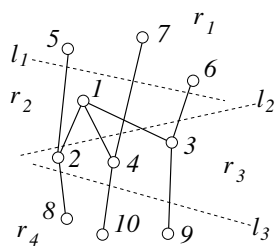

(c)

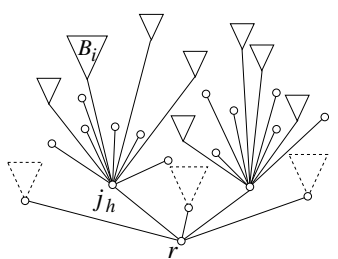

(d)

Fig. 1. (a) A tree $T_{u}$. (b)-(c) A level nonplanar tree and a region-level nonplanar tree whose underlying tree is $T_{u}$. (d) A schematization of $\mathcal{T}$. Joints and stabilizers are small circles. A solid triangle represents a branch, while a dashed triangle represents the subtree connected to a joint.

point in $r_{i}$ and a point in $r_{h}$, with $1 \leq i<h \leq k+1$, cuts all and only the segments $l_{i}, l_{i+1}, \ldots, l_{h-1}$, in this order. A drawing of a $k$-level tree $T=(V, E, \phi)$ is a regionlevel drawing if each vertex $v \in V$ such that $\phi(v)=i$ lies inside region $r_{i}$. A $k$-level tree is region-level nonplanar if it does not admit any planar region-level drawing. The 4-level tree $T$ whose underlying tree is shown in Fig. 1]a) is level nonplanar [10] (see Fig.1(b)). We show that $T$ is also region-level nonplanar (see Fig.1(c)).

Lemma 1. The 4-level tree $T$ whose underlying tree is shown in Fig. 1 a) is regionlevel nonplanar.

Lemma 1 will be vital for proving that a tree $\mathcal{T}$ and a path $\mathcal{P}$ exist not admitting any GSE. In fact, $\mathcal{T}$ contains many copies of the underlying tree of $T$, while $\mathcal{P}$ connects vertices in such a way to create the regions satisfying the above conditions and to enforce at least one of such copies to lie inside them according to the nonplanar leveling.

\section{The Counterexample}

In this section we describe a tree $\mathcal{T}$ and a path $\mathcal{P}$ not admitting any GSE.

The tree $\mathcal{T}$ has a root $r$ and $q$ vertices $j_{1}, \ldots, j_{q}$ at distance 1 from $r$, called joints. Each joint is connected to $l:=(s-1)^{4} \cdot 3^{2} \cdot x$ vertices of degree 1 , called stabilizers and to $x$ subtrees $B_{i}, i=1, \ldots, x$, called branches, each one consisting of a root $r_{i}$, $(s-1) \cdot 3$ vertices of degree $(s-1)$ adjacent to $r_{i}$, and $(s-2) \cdot(s-1) \cdot 3$ leaves at distance 2 from $r_{i}$. See Fig. 1(d). Vertices of the branches are called $B$-vertices and denoted by 1-, 2-, or 3-vertices, according to their distance from their joint.

For the sake of readability, we use variables $q, s$, and $x$ as parameters describing the size of certain structures, that will be given a value when the technical details are described. We claim that a number $n \geq\left(\begin{array}{c}2^{7} \cdot 3 \cdot x+2 \\ 3\end{array}\right)$ of vertices suffices for the counterexample. Despite the oversized number of vertices, tree $\mathcal{T}$ has limited height, that is, every vertex is at distance from the root at most 4 . This leads to the following property.

Property 1. Any simple path of tree edges starting at the root has at most 3 bends.

Path $\mathcal{P}$ is given by describing some basic recurring subpaths on the vertices of $\mathcal{T}$. The idea is to partition the set of branches adjacent to each joint into subsets of $s$ branches 
each and to connect the vertices of each set with path edges, according to some features of the tree structure, so defining the first building block, called cell. Then, cells belonging to the same joint are connected to create formations, for which we can ensure some properties on the intersection between tree and path edges. Further, formations are connected to create extended formations, which, in their turn, are connected to create sequences of extended formations. These structures are constructed in such a way that there exists a set of cells, connected to the same joint and being part of the same formation or extended formation, such that any four of these cells contain a copy of a region-level nonplanar tree, where the level of a vertex is determined by the cell it belongs to. Proving that four of such cells lie in different regions satisfying the properties of separation described above is equivalent to proving the existence of a crossing in $\mathcal{T}$. This allows us not to deal with single copies of the region-level nonplanar tree.

In the following we define such structures more formally and state their properties.

The most basic structure is defined by looking at how $\mathcal{P}$ connects the vertices of a set of $s$ branches connected to the same joint of $\mathcal{T}$. For each joint $j_{h}, h=1, \ldots, q$, and for each disjoint subset of $s$ branches $B_{i}, i=1, \ldots, s$, connected to $j_{h}$, we construct a set of $s$ cells as follows. For each $i=1, \ldots, s$, a cell $c_{i}(h)$ is composed of its head, its tail, and number $t$ of stabilizers. The head of $c_{i}(h)$ consists of the unique 1-vertex of $B_{i}$, the first three 2-vertices of each branch $B_{k}$, with $1 \leq k \leq s$ and $k \neq i$, that are not already used in a cell $c_{a}(h)$ with $1 \leq a<r$ and, for each 2-vertex not in $c_{i}(h)$ and not in $B_{i}$, the first 3-vertices not already used in a cell $c_{a}(h)$, with $1 \leq a<i$. The tail of $c_{i}(h)$ is created by considering a set of $3 \cdot s \cdot(s-1)^{2}$ branches adjacent to $j_{h}$, partitioned into $3 \cdot(s-1)^{2}$ subsets of $s$ subtrees each. The vertices of each subset are distributed between the cells in the same way as for the vertices of the head. Path $\mathcal{P}$ visits the vertices of a cell $c_{i}(h)$ as follows: Starting at the unique 1-vertex of the head, it reaches the 2-vertices of the head, then the 3-vertices of the head, then the 2vertices of the tail, and finally the 3 -vertices of the tail. After each occurrence of a 2- or 3 -vertex of the head, $\mathcal{P}$ visits a 1 -vertex of the tail, and after each occurrence of a 2- or a 3 -vertex of the tail, it visits a stabilizer of joint $j_{h}$ (see Fig. 2 (a)). Note that each set of $s$ cells constructed starting from the same set of $s$ branches is such that each subset of size four contains a region-level nonplanar tree, where the levels correspond to the membership of the vertices to a cell. Namely, consider four cells $c_{1}, \ldots, c_{4}$ belonging to the same set, leveled in this order. A region level nonplanar tree as in Fig. 1 consists of the 1-vertex $v$ of the head of $c_{2}$, the three 2-vertices of $c_{3}$ connected to $v$ and, for each of them, the 3-vertex of $c_{1}$ and the 3-vertex of $c_{4}$ connected to it.

The next structure describes how cells from four different sets are connected each other. A formation $F(H)$, where $H=\left(h_{1}, h_{2}, h_{3}, h_{4}\right)$ is a 4-tuple of indices of joints, consists of 592 cells. Namely, for each joint $j_{h_{i}}, 1 \leq i \leq 4, F(H)$ contains 148 cells of the same set of $s$ cells connected to $j_{h_{i}}$. Path $\mathcal{P}$ connects these cells in the order $\left(\left(h_{1} h_{2} h_{3}\right)^{37} h_{4}^{37}\right)^{4}$, that is, $\mathcal{P}$ repeats four times the following sequence: It connects $c_{1}\left(h_{1}\right)$ to $c_{1}\left(h_{2}\right)$, then to $c_{1}\left(h_{3}\right)$, then to $c_{2}\left(h_{1}\right)$, and so on until $c_{37}\left(h_{3}\right)$, from which it connects to $c_{1}\left(h_{4}\right)$, to $c_{2}\left(h_{4}\right)$, and so on till $c_{37}\left(h_{4}\right)$ (see Fig.2(b)). Since cells of $F(H)$ connected to the same joint belong to the same set of $s$ cells, the following holds:

Property 2. For any formation $F(H)$ and any joint $j_{h}$, with $h \in H$, if four cells $c_{r}(h) \in$ $F(H)$ are pairwise separated by straight lines, then there exists a crossing in $\mathcal{T}$. 


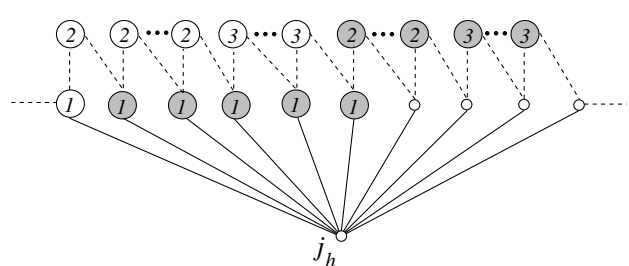

(a)

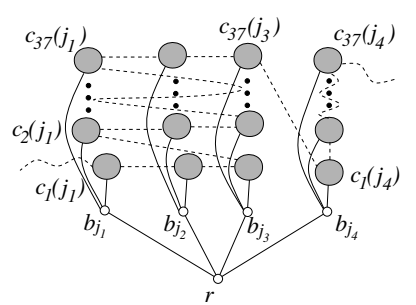

(b)

Fig. 2. (a) A cell. Vertices of the head are white and vertices of the tail are grey. $B$-vertices are large and stabilizers are small circles. (b) A formation.

Formations are connected by $\mathcal{P}$ to create an extended formation $\operatorname{EF}(H)$, where $H=$ $\left(H_{1}=\left(h_{1}, \ldots, h_{4}\right), H_{2}=\left(h_{5}, \ldots, h_{8}\right), \ldots, H_{x}=\left(h_{4 x-3}, \ldots h_{4 x}\right)\right)$ is an $x$-tuple of 4-tuples of disjoint indices of joints. For each 4-tuple $H_{i}, E F(H)$ contains $y-\frac{y}{x}$ formations $F_{1}\left(H_{i}\right), \ldots, F_{y-\frac{y}{x}}\left(H_{i}\right)$ not belonging to any other extended formation and composed of cells of the same set of $s$ cells connected to the same joint. Formations inside $E F(H)$ are connected in $\mathcal{P}$ in the order $\left(H_{1}, H_{2}, \ldots, H_{x}\right)^{y}$, that is, $\mathcal{P}$ connects $F_{1}\left(H_{1}\right)$ to $F_{1}\left(H_{2}\right)$, then to $F_{1}\left(H_{3}\right)$, and so on until $F_{1}\left(H_{x}\right)$, then to $F_{2}\left(H_{1}\right)$, to $F_{2}\left(H_{2}\right)$, and so on until $F_{y-\frac{y}{x}}\left(H_{x}\right)$. However, in each of these $y$ repetitions one $H_{i}$ is missing. Namely, in the $k$-th repetition $\mathcal{P}$ does not reach any formation at $H_{m}$, with $m=k \bmod x$. We say that the $k$-th repetition has a defect at $m$ and a subsequence $\left(H_{1}, H_{2}, \ldots, H_{x}\right)^{x}$ is a full repetition, having exactly one defect at each tuple.

Extended formations are connected by $\mathcal{P}$ in a sequence of extended formations $\operatorname{SEF}(H)$, where $H=\left(H_{1}^{*}, \ldots, H_{12}^{*}\right)$ is a 12 -tuple of $x$-tuples of 4 -tuples of disjoint indices of joints. For each $x$-tuple $H_{i}^{*}$, with $i=1, \ldots, 12$, there exist 110 extended formations $E F_{j}\left(H_{i}^{*}\right)$, with $j=1, \ldots, 110$, not belonging to any other sequence of extended formations, that are connected by $\mathcal{P}$ in the order $\left(H_{1}^{*}, \ldots, H_{12}^{*}\right)^{(120)}$. We have two types of sequences of extended formations. In the first type, in each repetition $\left(H_{1}^{*}, \ldots, H_{12}^{*}\right)$ one extended formation $E F\left(H_{m}\right)$ is missing, creating a defect at $m$. In the second type, in each repetition $\left(H_{1}^{*}, \ldots, H_{12}^{*}\right)$ two consecutive extended formations are missing. Namely, in the $k$-th repetition $\mathcal{P}$ skips the extended formations $E F\left(H_{m}^{*}\right)$ and $E F\left(H_{m+1}^{*}\right)$, with $m=k \bmod 12$, creating a double defect at $m$.

The size of $s$ can now be fixed as the number of formations in an extended formation times the number of cells in a formation, that is, $s:=\left(y-\frac{y}{x}\right) \cdot 37 \cdot 4$. Further, $q:=48 x$, as we need 4 sequences of extended formations (of size 12 each) not sharing any joint. We claim that $x=7 \cdot 3^{2} \cdot 2^{23}$ and $y=7^{2} \cdot 3^{3} \cdot 2^{26}$ is sufficient in the proofs.

\section{Overview}

In this section we present the main arguments leading to the conclusion that $\mathcal{T}$ and $\mathcal{P}$ do not admit any GSE. The main idea is to use the structures given by $\mathcal{P}$ to fix a part of $\mathcal{T}$ in a specific shape creating restrictions for the placement of the further substructures attached to it. Then, we show that such restrictions lead to a crossing in any possible GSE of $\mathcal{P}$ and $\mathcal{T}$. In the following, we will perform an analysis of the geometrical properties of each possible embedding, in order to show that none of them is feasible. 
First, we give some definitions and properties of cells enforced by properties of region-level planar drawings and by the order of the cells inside a formation.

Let $c_{1}(h), c_{2}(h)$ be two cells connected to a joint $j_{h}$ that can not be separated by a straight line and a cell $c^{\prime}\left(h^{\prime}\right)$ connected to a joint $j_{h^{\prime}}$, with $h^{\prime} \neq h$. A passage $P$ between $c_{1}, c_{2}$, and $c^{\prime}$ exists if the path of $c^{\prime}$ separates vertices of $c_{1}$ from vertices of $c_{2}$ (see Fig. 3 (a)). Since the separation can not be straight, there is a vertex of $c^{\prime}$ lying inside the convex hull of the vertices of $c_{1} \cup c_{2}$. Hence, there exist at least two path-edges $e_{1}, e_{2}$ of $c^{\prime}$ intersected by tree-edges connecting vertices of $c_{1}$ to vertices of $c_{2}$.

For two passages $P_{1}$ between $c_{1}\left(h_{1}\right), c_{2}\left(h_{1}\right)$, and $c^{\prime}\left(h_{1}^{\prime}\right)$, and $P_{2}$ between $c_{3}\left(h_{2}\right)$, $c_{4}\left(h_{2}\right)$, and $c^{\prime}\left(h_{2}^{\prime}\right)$ (w.1.o.g., assume $h_{1}<h_{1}^{\prime}, h_{2}<h_{2}^{\prime}$, and $h_{1}<h_{2}$ ), we distinguish three configurations: (i) If $h_{1}^{\prime}<h_{2}, P_{1}$ and $P_{2}$ are independent; (ii) if $h_{2}^{\prime}<h_{1}^{\prime}, P_{2}$ is nested into $P_{1}$; and (iii) if $h_{2}<h_{1}^{\prime}<h_{2}^{\prime}, P_{1}$ and $P_{2}$ are interconnected (see Fig. 3 (b)).

Let $c_{1}(h), c_{2}(h)$, and $c^{\prime}\left(h^{\prime}\right)$ be three cells creating a passage. We call a door any triangle given by a vertex $v^{\prime}$ of $c^{\prime}$ inside the convex hull of $c_{1} \cup c_{2}$ and by any two vertices of $c_{1} \cup c_{2}$ that encloses neither any other vertex of $c_{1}, c_{2}$ nor any vertex of $c^{\prime}$ that is closer than $v^{\prime}$ to $j_{h^{\prime}}$ in $\mathcal{T}$. A door is open if no tree-edge incident to $v^{\prime}$ crosses the side of the triangle between the vertices of $c_{1}$ and $c_{2}$, otherwise it is closed.

Consider two joints $j_{a}$ and $j_{b}$, with $j_{h}, j_{a}, j_{h}^{\prime}, j_{b}$ in this circular order around the root. Any polyline connecting the root to $j_{a}$, then to $j_{b}$, and again to the root, without crossing tree edges, must traverse each door by crossing both the sides adjacent to $v^{\prime}$. If a door is closed, such a polyline has to bend between the two sides adjacent to $v^{\prime}$. In the rest of the argument we will exploit this fact to obtain the claimed property that a large part of $\mathcal{T}$ has to follow the same shape. In view of this, we state the following lemmata.

Lemma 2. For each formation $F(H)$, with $H=\left(h_{1}, \ldots, h_{4}\right)$, there exists a passage between some cells $c_{1}\left(h_{a}\right), c_{2}\left(h_{a}\right), c^{\prime}\left(h_{b}\right) \in F(H)$, with $1 \leq a, b \leq 4$.

\section{Lemma 3. Each passage contains at least one closed door.}

Hence, each formation contains at least one closed door. In the following we prove that the combined effects of closed doors of different formations enforces more restrictions on the shape of the tree. First, we exploit the Ramsey Theorem [15] to state that there exists a set of joints such that any two joints contain cells creating a passage.

Lemma 4. Given a set of joints $J=\left\{j_{1}, \ldots, j_{y}\right\}$, with $|J|=y:=\left(\begin{array}{c}2^{7} \cdot 3 \cdot x+2 \\ 3\end{array}\right)$, there exists a subset $J^{\prime}=\left\{j_{1}^{\prime}, \ldots, j_{r}^{\prime}\right\}$, with $\left|J^{\prime}\right|=r \geq 2^{7} \cdot 3 \cdot x$, such that for each pair of joints $j_{i}^{\prime}, j_{h}^{\prime} \in J^{\prime}$ there exist two cells $c_{1}(i), c_{2}(i)$ creating a passage with a cell $c^{\prime}(h)$.

Consider two paths $p_{1}=\left\{u_{1}, v_{1}, w_{1}\right\}$ and $p_{2}=\left\{u_{2}, v_{2}, w_{2}\right\}$. The bendpoint $v_{1}$ of $p_{1}$ encloses the bendpoint $v_{2}$ of $p_{2}$ if $v_{2}$ is internal to triangle $\triangle\left(u_{1}, v_{1}, w_{1}\right)$.

Consider a set of joints $J=\left\{j_{1}, \ldots, j_{k}\right\}$ in clockwise order around the root. The channel $c_{i}$ of a joint $j_{i}$, with $i=2, \ldots, k-1$, is the region given by the pair of paths, one path of $j_{i-1}$ and one path of $j_{i+1}$, with the maximum number of enclosing bendpoints with each other. We say that $c_{i}$ is an $x$-channel if the number of enclosing bendpoints is $x$. Note that, by Prop. 1, $x \leq 3$. See Fig. 3 (c). An $x$-channel $c_{i}$ is composed of $x+1$ channel segments. The first channel segment $c s_{1}$ is the part of $c_{i}$ visible from the root. The $h$-th channel segment $c s_{h}$ is the part of $c_{i}$ disjoint from $c s_{h-1}$ that is bounded by 


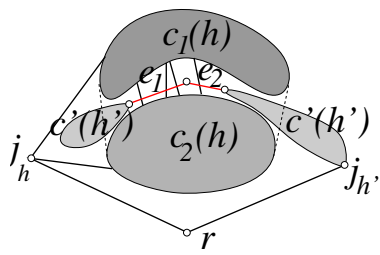

(a)

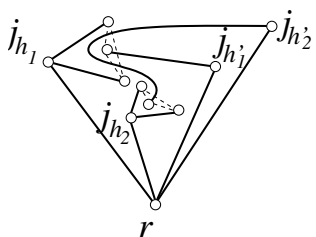

(b)

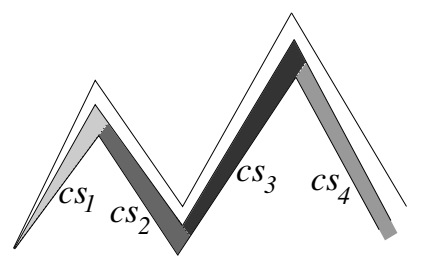

(c)

Fig. 3. (a) A passage between cells $c_{1}, c_{2}$, and $c^{\prime}$. (b) Two interconnected passages. (c) A 3channel and its channel segments.

the elongations of the paths of $j_{i-1}$ and $j_{i+1}$ after the $h$-th bend. The bending area $b(a, a+1)$ of $c_{i}$ is the region visible from all the points of $c s_{a}$ and $c s_{a+1}$. As channels are created by tree-edges, only path-edges can cross the boundaries of the channel.

We study the relationships between path-edges and channels. As every second vertex of $\mathcal{P}$ in a cell is either a 1-vertex or a stabilizer, we have the following property.

Property 3. For any path-edge $(a, b)$, at least one of $a$ and $b$ lies inside either $c s_{1}$ or $c s_{2}$.

A blocking cut is a path edge connecting two consecutive channel segments by cutting some of the other channels twice.

Property 4. If a channel that is cut twice by a blocking cut has vertices in both the channel segments cut by the path-edge, then it has vertices in a different channel segment.

Next, based on Prop. 4 we show that any set of joints as in Lemma 4 contains a subset of joints creating interconnected passages such that each pair of paths of tree-edges starting at the root and containing such joints has at least two common enclosing bendpoints, which implies that most of them create 2-channels. From now on, we identify a joint with the channel it belongs to. Then, when dealing with a passage between two cells $c_{1}(h), c_{2}(h)$ of a joint $j_{h}$ and a cell $c^{\prime}\left(h^{\prime}\right)$ of a joint $j_{h^{\prime}}$, we might also say that there is a passage between joints $j_{h}$ and $j_{h}^{\prime}$ or between the corresponding channels.

Lemma 5. Let $J=\left\{j_{1}, \ldots, j_{k}\right\}$ be a set of joints such that there exists a passage between each pair $\left(j_{i}, j_{h}\right)$, with $1 \leq i, h \leq k$. Let $\mathcal{P}_{1}=\left\{P \mid P\right.$ connects $c_{i}$ and $c_{\frac{3 k}{4}+1-i}$, for $\left.i=1, \ldots, \frac{k}{4}\right\}$ and $\mathcal{P}_{2}=\left\{P \mid P\right.$ connects $c_{\frac{k}{4}+i}$ and $c_{k+1-i}$, for $\left.i=1, \ldots, \frac{k}{4}\right\}$ be two sets of passages between pairs of joints in J (see Fig. $4(a)$ ). Then, for at least $\frac{k}{4}$ of the joints of one set of passages, say $\mathcal{P}_{1}$, there exist paths in $\mathcal{T}$, starting at the root and containing these joints, which traverse all the doors of $\mathcal{P}_{2}$ with at least 2 and at most 3 bends. Also, at least half of these joints create an $x$-channel, with $2 \leq x \leq 3$.

By Lemma 5, any formation attached to a certain subset of joints must use at least three different channel segments. In the remainder we focus on this subset and give some properties holding for it. As we need a full sequence of extended formations attached to these joints, $k$ has to be at least eight times the number of channels inside a sequence of extended formations, that is, $k \geq 8 \cdot 48 x=2^{7} \cdot 3 x$.

A formation $F$ is nested in a formation $F^{\prime}$ if there exist four path-edges $e_{1}, e_{2} \in F$ and $e_{1}^{\prime}, e_{2}^{\prime} \in F^{\prime}$ cutting a boundary $c b$ of a channel $c$ such that all the vertices of the 


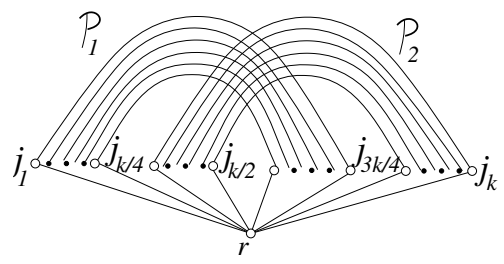

(a)

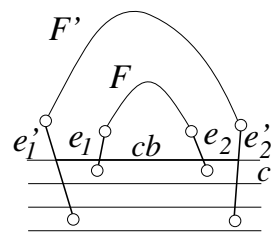

(b)

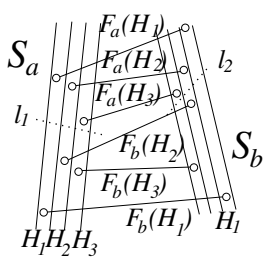

(c)

Fig. 4. (a) Two sets of passages $\mathcal{P}_{1}$ and $\mathcal{P}_{2}$ as described in Lemma 5. (b) A formation $F$ nested in a formation $F^{\prime}$. (c) Two independent sets $S_{a}$ and $S_{b}$.

path in $F$ between $e_{1}$ and $e_{2}$ lie inside the region delimited by $c b$ and by the path in $F^{\prime}$ between $e_{1}^{\prime}$ and $e_{2}^{\prime}$ (see Fig.4(b)). A series of pairwise nested formations $F_{1}, \ldots, F_{k}$ is $r$-nested if there exist $r$ formations $F_{q_{1}}, \ldots, F_{q_{r}}$, with $1 \leq q_{1}, \ldots, q_{r} \leq k$, such that the 4-tuples of $F_{q_{1}}, \ldots, F_{q_{r}}$ have at least one common joint $j$, and such that for each pair $F_{q_{p}}, F_{q_{p+1}}$ there exists at least one formation $F_{z}$, with $1 \leq z \leq k$, such that the 4-tuple of $F_{z}$ does not contain $j, F_{q_{p}}$ is nested in $F_{z}$ and $F_{z}$ is nested in $F_{q_{p+1}}$.

Let $S_{1}, \ldots, S_{k}$ be sets of formations of one extended formation $E F(H)$ such that each set $S_{i}$, for $i=1, \ldots, k$, contains the formations $F_{i}\left(H_{1}\right), \ldots, F_{i}\left(H_{r}\right)$, such that $\left(H_{1}, \ldots, H_{r}\right) \subset H$. Let $F_{a}\left(H_{c}\right)$ be not nested in $F_{b}\left(H_{d}\right)$, for each $1 \leq a, b \leq k, a \neq b$, and $1 \leq c, d \leq r$. If for each two sets $S_{a}, S_{b}$ there exists a line $l_{1}$ (a line $l_{2}$ ) separating the vertices of $S_{a}$ (of $S_{b}$ ) inside channel segment $c s_{1}$ (channel segment $c s_{2}$ ), then sets $S_{1}, \ldots, S_{k}$ are independent (see Fig. 4 (c)).

In the following lemmata we prove that in any extended formation there exists a nesting of a certain depth (Lemma 8 ) by first proving that in any extended formation the number of independent sets of formations is limited (Lemma 6) and then by showing that, although some formations might be neither nested nor independent, there exists a certain number that have to be either independent or nested (Lemma 7).

Lemma 6. No extended formation contains $n \geq 2^{22} \cdot 14$ independent sets offormations $S_{1}, \ldots, S_{n}$ such that each set $S_{i}$ contains formations $F_{i}\left(H_{1}\right), \ldots, F_{i}\left(H_{r}\right)$, with $r \geq 22$.

Lemma 7. Let $E F$ be an extended formation and let $Q_{1}, \ldots, Q_{4}$ be four subsequences of formations, each consisting of a whole repetition $\left(H_{1}, H_{2}, \ldots, H_{x}\right)$ of EF. Then, there exists either a pair of nested subsequences or a pair of independent subsequences.

Lemma 8. For every extended formation EF, there exists a $k$-nesting, with $k \geq 6$.

Then, we study how such a nesting can be performed inside the channels. We will conclude that, in any possible shape of the tree, either it is not possible to draw the nesting formations planar, or that any planar drawing of such formations induces further geometrical constraints not allowing a planar drawing of the rest of the tree.

Let $c s_{a}$ and $c s_{b}$, with $1 \leq a, b \leq 4$, be two channel segments. If the elongation of $c s_{a}$ intersects $c s_{b}$, then it is possible to connect from $c s_{b}$ to $c s_{a}$ by cutting both the sides of $c s_{a}$. In this case, $c s_{a}$ and $c s_{b}$ have a 2-side connection (see Fig. 5b)). On the contrary, if the elongation of $c s_{a}$ does not intersect $c s_{b}$, only one side of $c s_{a}$ can be used. In this case, $c s_{a}$ and $c s_{b}$ have a 1 -side connection (see Fig. 5]a)). 
First, we consider the case in which only 1-side connections are possible (Fig. 3 (c)). We prove that, in this configuration, the existence of a nesting in one extended formation results in a crossing in either $\mathcal{T}$ or $\mathcal{P}$.

Proposition 1. If every two channel segments have a 1 -side connection, then $\mathcal{T}$ and $\mathcal{P}$ do not admit any GSE.

Next, we study the case in which there exist 2-side connections. We distinguish two types of 2-side connections. If the elongation of channel segment $c s_{a}$ intersecting channel segment $c s_{b}$ starts at the bendpoint that is closer to the root, we have a low Intersection $I_{(a, b)}^{l}$ (see Fig.5 ( $\left.\mathrm{c}\right)$ ). Otherwise, we have a high Intersection $I_{(a, b)}^{h}$ (see Fig.5(d)). We use notation $I_{(a, b)}$ to describe both $I_{(a, b)}^{h}$ and $I_{(a, b)}^{l}$. Two intersections $I_{(a, b)}$ and $I_{(c, d)}$ are disjoint if $a, d \in\{1,2\}$ and $b, c \in\{3,4\}$. E.g., $I_{(1,3)}$ and $I_{(4,2)}$ are disjoint, while $I_{(1,3)}$ and $I_{(2,4)}$ are not. Since consecutive channel segments can not create 2 -side connections, to explore all the possible shapes we consider the combinations of low and high intersections of channel segments $c s_{1}$ and $c s_{2}$ with $c s_{3}$ and $c s_{4}$. First, we prove that intersections of adjacent channels have to maintain certain consistencies.

Lemma 9. Consider two channels $c h_{p}, c h_{q}$ with the same intersections $I_{(a, b)}$. Then, none of channels $c h_{i}$, where $p<i<q$, have an intersection that is disjoint with $I_{(a, b)}$.

As for Proposition 1 in order to prove that 2-side connections are not sufficient, we exploit the existence of the nesting shown in Lemma 8 . Note that every extended formation using a channel segment $c s_{a}$ to place the nesting must place vertices inside the adjacent bending area. We prove that not many of the nesting formations can use the part of the path that creates the nesting to place vertices in such a bending area.

Lemma 10. Consider a nesting offormations inside a sequence of extended formations on an intersection $I_{(a, b)}$, with $a \leq 2$. Then, one of the formations contains a pair of path-edges $(u, v),(v, w)$, with $v$ lying inside channel segment $c s_{a}$, separating some formations in $c s_{a}$ from bending area $b(a, a+1)$ or $b(a-1, a)$ (see Fig. 5 e e)).

Let the inner area and outer area of $c s_{a}$ be the two parts in which $c s_{a}$ is split by edges $(u, v),(v, w)$, as in Lemma 10 . Since in every extended formation a path exists connecting the inner and the outer area by going around either $u$ or $w$, the extended formations using such paths create a structure that is analogous to a nesting of formations. We prove that, as every repetition of an extended formation contains a defect, if only 1-side connections are available to host such paths, then a crossing in $\mathcal{T}$ or $\mathcal{P}$ is created.

Lemma 11. Let $c s_{a}$ be a channel segment that is split into inner area and outer area by two edges such that every extended formation of a sequence of extended formations has vertices in both the areas. If the only possibility to connect vertices from the inner to the outer area is with a 1 -side connection, then $\mathcal{T}$ and $\mathcal{P}$ do not admit any GSE.

Lemma11 states that one single 2-side connection is not sufficient to obtain a GSE of $\mathcal{T}$ and $\mathcal{P}$. We prove that a further 2-side connection is not useful if it is not disjoint.

Proposition 2. If there exists no pair of disjoint 2-side connections, then $\mathcal{T}$ and $\mathcal{P}$ do not admit any GSE.

Note that it is sufficient to restrict the analysis to cases $I_{(1,3)}$ (see Figs. 6(a)-(b)) and $I_{(3,1)}$, as the cases involving 2 and 4 can be reduced to them. 


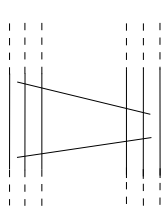

(a)

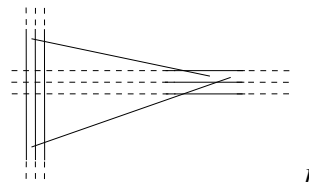

(b)

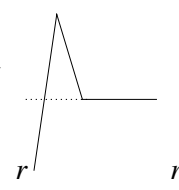

(c)

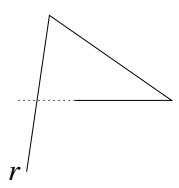

(d)

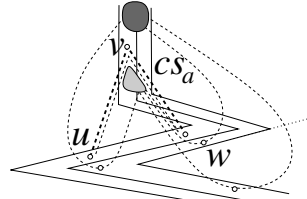

(e)

Fig. 5. (a) A 1-side connection. (b) A 2-side connection. (c) A low Intersection. (d) A high Intersection. (e) A situation as in Lemma 10 Inner and the outer areas are represented by a light grey and a dark grey region, respectively.

Lemma 12. If a shape contains an intersection $I_{(1,3)}$ and does not contain any other intersection that is disjoint with $I_{(1,3)}$, then $\mathcal{T}$ and $\mathcal{P}$ do not admit any GSE.

Lemma 13. If there exists a sequence of extended formations in any shape containing an intersection $I_{(3,1)}$, then $\mathcal{T}$ and $\mathcal{P}$ do not admit any GSE.

Finally, we tackle the general case where two disjoint intersections exist.

Proposition 3. If there exists two disjoint 2-side connections, then $\mathcal{T}$ and $\mathcal{P}$ do not admit any GSE.

Note that Lemma 13 stated a property that is stronger than Proposition 2 In fact, a GSE cannot be obtained in any shape containing $I_{(3,1)}$, even if a disjoint intersection is present. Hence, we only consider the eight configurations with $I_{(1,3)}$ and $I_{(4,\{1,2\})}^{h, l}$.

Let $c s_{i}$ and $c s_{i+1}$ be two consecutive channel segments of a channel $c h_{k}$ and let $e$ be a path-edge crossing the boundary of one of $c s_{i}$ and $c s_{i+1}$, say $c s_{i}$. Edge $e$ is a double cut at $c h_{k}$ if the line through $e$ cuts $c h_{k}$ in $c s_{i+1}$. A double cut is simple if the elongation of $e$ cuts $c s_{i+1}$ (see Fig. 6(c)) and non-simple if $e$ itself cuts $c s_{i+1}$ (see Fig. 6(d)). A double cut of an extended formation $E F$ is extremal at bending area $b(i, i+1)$ if no double cut of $E F$ closer to $b(i, i+1)$ exists. Double cut $e$ blocks visibility to $b(i, i+1)$ for a part of $c s_{i}$ in each channel $c h_{h}$ with $h>k$ or with $h<k$.

We show that a certain ordering of extremal double cuts in two consecutive channel segments leads to a non-planarity, and that, because of the double defect in every repetition of an extended formation, both shapes $I_{(1,3)}^{h} I_{(4,2)}^{h, l}$ induce this order (Lemma 16).

Lemma 14. Let $c s_{i}$ and $c s_{i+1}$ be two consecutive channel segments. If there exists an ordered set $S:=(1,2, \ldots, 5)^{3}$ of extremal double cuts cutting $c s_{i}$ and $c s_{i+1}$ such that the order of the intersections of the double cuts with $c s_{i}$ (with $c s_{i+1}$ ) is coherent with the order of $S$, then $\mathcal{T}$ and $\mathcal{P}$ do not admit any GSE.

We first show that double cuts exist in $I_{(1,3)}^{h} I_{(4,2)}^{h}$. This is easy to see in $I_{(1,3)}^{h} I_{(4,2)}^{l}$.

Lemma 15. Shape $I_{(1,3)}^{h} I_{(4,2)}^{h}$ creates double cuts in at least one bending area.

Lemma 16. Every sequence of extending formations in shape $I_{(1,3)}^{h} I_{(4,2)}^{h, l}$ contains an ordered set $(1,2, \ldots, 5)^{3}$ of extremal double cuts at either $b(2,3)$ or $b(3,4)$. 


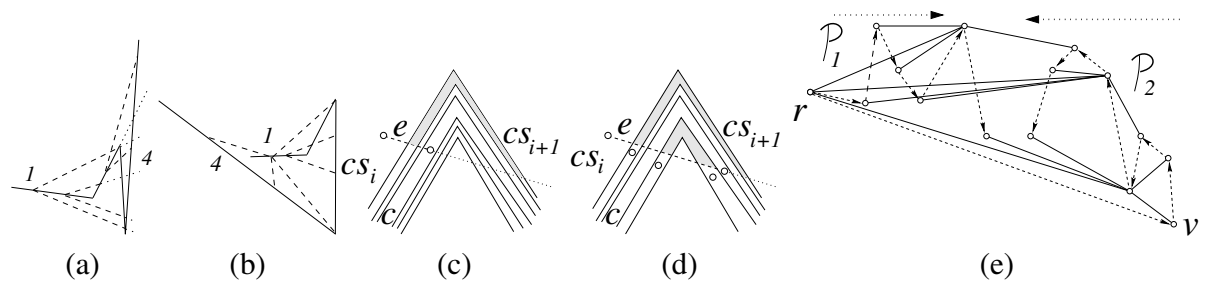

(a)

(b)

(c)

(d)

(e)

Fig. 6. (a) Case $I_{(1,3)} I_{(2,4)}^{h}$. As a nesting at $I_{(1,3)}$ must reach $b(2,3)$, it crosses any nesting at $I_{(2,4)}^{h}$. (b) Case $I_{(1,3)} I_{(2,4)}^{l}$. A nesting at $I_{(1,3)}$ crosses any nesting at $I_{(2,4)}^{l}$ and any extended formation nesting at $I_{(1,4)}$ creates a nesting at $I_{(1,3)}$, as it must reach $b(2,3)$ and $b(3,4)$. (c) A simple double cut. (d) A non-simple double cut. (e) Construction of a GSE of a height- 2 tree and a path. Arrows indicate that the subpaths before and after $r$ are monotone in opposite directions.

Finally, we consider shapes $I_{(1,3)}^{l} I_{(4,2)}^{h, l}$. Note that, in both cases, $c s_{2}$ lies on the convex hull of the shape. We show that this yields a crossing either in $\mathcal{T}$ or in $\mathcal{P}$.

Lemma 17. If $c s_{2}$ is part of the convex hull, then $\mathcal{T}$ and $\mathcal{P}$ do not admit any GSE.

Based on the above discussion, we state the following theorem.

Theorem 1. There exist a tree and a path that do not admit any GSE.

\section{Constructing a GSE of a Tree of Height 2 and a Path}

In this section we sketch the algorithm for constructing a GSE of a tree $\mathcal{T}$ of height 2 and a path $\mathcal{P}$. See Fig. 6(e). Draw the root $r$ of $\mathcal{T}$ as the leftmost vertex. Consider the two subpaths $\mathcal{P}_{1}$ and $\mathcal{P}_{2}$ of $\mathcal{P}$ starting at $r$. Assign an orientation to $\mathcal{P}_{1}$ (to $\mathcal{P}_{2}$ ) such that $r$ is the only source of $\mathcal{P}_{1}$ (of $\mathcal{P}_{2}$ ). Draw $\mathcal{P}_{1}$ to the right of $r$, placing its vertices from the left to the right following the orientation of $\mathcal{P}_{1}$. Draw the vertex $v$ following $r$ in $\mathcal{P}_{2}$ as the rightmost vertex. Finally, draw $\mathcal{P}_{2}$ to the left of $v$, placing its vertices from the right to the left following the orientation of $\mathcal{P}_{2}$, so that the leftmost vertex of $\mathcal{P}_{2}$ is to the right of the rightmost vertex of $\mathcal{P}_{1}$.

\section{Conclusions}

In this paper we have shown that there exist a tree $\mathcal{T}$ and a path $\mathcal{P}$ on the same set of vertices that do not admit any GSE. We first extended the concept of level nonplanar trees [10] to the one of region-level nonplanar trees, and showed that there exist trees not admitting any planar embedding if the vertices are forced to lie inside certain regions according to a prescribed ordering. Then, we constructed $\mathcal{T}$ and $\mathcal{P}$ so that $\mathcal{P}$ creates these regions and enforces at least one of the many region-level nonplanar trees composing $\mathcal{T}$ to lie inside them in the desired order. Our result implies that two edge-disjoint trees exist not admitting any GSE, answering a question posed in [14].

Note that, despite the huge number of vertices, $\mathcal{T}$ can be considered as "simple", as its height is just 4 . In this direction, we proved that any tree of height 2 admits a GSE with any path, giving raise to an intriguing question: What about a tree of height 3 ? 


\section{References}

1. Angelini, P., Geyer, M., Kaufmann, M., Neuwirth, D.: On a tree and a path with no geometric simultaneous embedding. Tech. Report 176, Dipartimento di Informatica e Automazione, Roma Tre University (2010)

2. Brandes, U., Erten, C., Fowler, J., Frati, F., Geyer, M., Gutwenger, C., Hong, S.H., Kaufmann, M., Kobourov, S., Liotta, G., Mutzel, P., Symvonis, A.: Colored simultaneous geometric embeddings. In: Lin, G. (ed.) COCOON 2007. LNCS, vol. 4598, pp. 254-263. Springer, Heidelberg (2007)

3. Brass, P., Cenek, E., Duncan, C., Efrat, A., Erten, C., Ismailescu, D., Kobourov, S., Lubiw, A., Mitchell, J.: On simultaneous planar graph embeddings. Comp. Geom. 36(2), 117-130 (2007)

4. Cabello, S., van Kreveld, M., Liotta, G., Meijer, H., Speckmann, B., Verbeek, K.: Geometric simultaneous embeddings of a graph and a matching. In: Eppstein, D., Gansner, E.R. (eds.) GD 2009. LNCS, vol. 5849, pp. 183-194. Springer, Heidelberg (2010)

5. Di Giacomo, E., Didimo, W., van Kreveld, M., Liotta, G., Speckmann, B.: Matched drawings of planar graphs. J. Graph Alg. Appl. 13(3), 423-445 (2009)

6. Erten, C., Kobourov, S.G.: Simultaneous embedding of planar graphs with few bends. J. Graph Alg. Appl. 9(3), 347-364 (2005)

7. Estrella-Balderrama, A., Fowler, J., Kobourov, S.G.: Characterization of unlabeled level planar trees. Comp. Geom. 42(6-7), 704-721 (2009)

8. Fowler, J., Jünger, M., Kobourov, S.G., Schulz, M.: Characterizations of restricted pairs of planar graphs allowing simultaneous embedding with fixed edges. In: Broersma, H., Erlebach, T., Friedetzky, T., Paulusma, D. (eds.) WG 2008. LNCS, vol. 5344, pp. 146-158. Springer, Heidelberg (2008)

9. Fowler, J., Kobourov, S.: Characterization of unlabeled level planar graphs. In: Hong, S.H., Nishizeki, T., Quan, W. (eds.) GD 2007. LNCS, vol. 4875, pp. 37-49. Springer, Heidelberg (2008)

10. Fowler, J., Kobourov, S.: Minimum level nonplanar patterns for trees. In: Hong, S.-H., Nishizeki, T., Quan, W. (eds.) GD 2007. LNCS, vol. 4875, pp. 69-75. Springer, Heidelberg (2008)

11. Frati, F.: Embedding graphs simultaneously with fixed edges. In: Kaufmann, M., Wagner, D. (eds.) GD 2006. LNCS, vol. 4372, pp. 108-113. Springer, Heidelberg (2007)

12. Frati, F., Kaufmann, M., Kobourov, S.: Constrained simultaneous and near-simultaneous embeddings. J. Graph Alg. Appl. 13(3), 447-465 (2009)

13. Gassner, E., Jünger, M., Percan, M., Schaefer, M., Schulz, M.: Simultaneous graph embeddings with fixed edges. In: Fomin, F.V. (ed.) WG 2006. LNCS, vol. 4271, pp. 325-335. Springer, Heidelberg (2006)

14. Geyer, M., Kaufmann, M., Vrt'o, I.: Two trees which are self-intersecting when drawn simultaneously. Disc. Math. 309(7), 1909-1916 (2009)

15. Graham, R.L., Rothschild, B.L., Spencer, J.H.: Ramsey Theory. John Wiley \& Sons, Chichester (1990)

16. Halton, J.H.: On the thickness of graphs of given degree. Inf. Sc. 54(3), 219-238 (1991)

17. Pach, J., Wenger, R.: Embedding planar graphs at fixed vertex locations. Graphs and Comb. 17(4), 717-728 (2001)

18. Thomassen, C.: Embeddings of graphs. Disc. Math. 124(1-3), 217-228 (1994)

19. Tutte, W.T.: How to draw a graph. London Math. Society 13, 743-768 (1962) 